\title{
Primary Extranodal Non-Hodgkin's Lymphoma: Clinicopathological Features, Survival and Treatment Outcome in Two Cancer Centers of Southern Turkey
}

\author{
Huseyin Mertsoylu ${ }^{1 *}$,Sadik Muallaoglu ${ }^{1}$, Ayberk Ali Besen ${ }^{2}$, Suleyman Erdogdu $^{3}$, \\ Ahmet Sezer ${ }^{1}$, Ali Murat Sedef ${ }^{1}$, Fatih Kose ${ }^{1}$, Ali Arican ${ }^{3}$, Ozgur Ozyilkan'
}

\begin{abstract}
Background: The aim of this study was to assess the epidemiological and clinicopathological characteristics of primary extranodal non-Hodgkin's lymphoma (pENL) patients, focusing on treatment and survival outcome. Materials and Methods: Between October 2003 and March 2012, 802 patients with non-Hodgkin's lymphoma (NHL) were diagnosed and treated in two different cancer centers of Southern Turkey. Results: pENL, constituted $12.4 \%(100 / 802)$ of all NHL studied during this period. Median age of the patients was 56 years (range 17-87 years) and the male: female distribution was $3: 2$. Eighty-five of 100 patients $(85 \%)$ were in stage $I / I I, 9 / 100(9 \%)$ in stage III, whereas 6/100 $(6 \%)$ were in stage IV. Head and neck constituted the most common site $(51 / 100$, $51 \%$ ), followed by gastrointestinal tract (GIL) $(37 / 100,37 \%)$, and cerebrum (CL) $(5 / 100,5 \%)$. Diffuse large B cell lymphoma (DLBCL) was the most common histological type, observed in $53 \%$ of patients, followed by marginal zone extranodal lymphoma $(\mathbf{1 3 \%})$. Most of patients $(\mathbf{7 6 \%})$ received a CHOP containing regimen. Complete remission (CR) were achieved in $71 \%$ of patients. The median follow-up duration of all patients was reported as 37.6 months (range, 0.8-165 months). This period was reported as 137.5 months (range, 117.51578.6 months) in gastrointestinal lymphoma (GIL) patients, 119.0 months (range, 91.8-146.1 months) in head and neck lymphoma (HNL) patients, and 18.4 months (range, 12.6-24.1 months) in cerebral lymphoma (CL) patients. Conclusions: Head and neck, and the gastrointestinal tract were the two most common extranodal sites observed. Histologically DLBC accounted for the majority of cases. Most patients were on earlier stages, had low-low intermediate IPI scores and had a favorable prognosis.
\end{abstract}

Keywords: Extranodal lymphoma - non-Hodgkin's lymphoma - clinicopathological characteristics

Asian Pac J Cancer Prev, 15 (17), 7207-7211

\section{Introduction}

Most non-Hodgkin's Lymphomas (NHL) arise in lymph nodes or other lymphatic tissues such as the spleen, Waldeyer's ring and thymus. However, a substantial percentage of NHL arises from tissues other than lymph nodes and even from sites, which normally contain no lymphoid tissue. These forms are referred to as primary extranodal lymphomas (pENL). Approximately 25-40\% of NHL arises primarily from sites other than lymph nodes (d'Amore et al., 1991; Freeman et al., 1972; Otter et al., 1989; Newton et al., 1997; Padhi et al., 2012).

The definition of pENL, particularly in the presence of both nodal and extranodal disease, remains a controversial issue. Different criteria have been proposed by various authors in the past, to categorize these entities (Dawson et al., 1961; Krol et al., 2003). As per Dowson criteria, lymphoma is said to be primarily extranodal in 1) absence of palpable superficial lymph nodes on first physical examination; 2) absence of mediastinal lymphadenopathy detected on plain chest $\mathrm{x}$-ray; 3) dominant lesion at extranodal sites; 4) involvement of lymph nodes in the vicinity of the primary lesion; 5) white blood cell (WBC) count within normal range.

The incidence of extranodal NHL in western countries has increased substantially in the last 40 years (Groves et al., 2000; Chiu et al., 2003). This may partly be due to improved diagnostic procedures (particularly in brain and gastrointestinal lymphomas) and changes in classification systems, but much of the changes are real and the AIDS epidemic in the 1980's does not completely explain this rise (Groves et al., 2000).

The etiology of ENL's appears to be multifactorial and includes immune suppression, infections both viral and bacterial, and exposure to pesticides and other environmental agents. 
The aim of this study was to assess the epidemiological and clinicopathological characteristics of pENL patients, focusing on treatment and survival outcome.

\section{Materials and Methods}

Between October 2003 and March 2012, 802 patients with NHL were diagnosed and treated in two different centers (Mersin University, School of Medicine, Department of Medical Oncology and Baskent University School of Medicine, Department of Medical Oncology).

Data pertaining to patients' demography, clinical presentation, routine complete blood count (CBC), microbiological (HIV, HCV, and Hepatitis B status) and biochemical parameters [Serum total protein, serum albumin, serum urea, creatinine, uric acid, lactate dehydrogenase (LDH) bilirubin and liver enzymes] were obtained from medical records. Clinical stage was defined according to the Ann Arbor classification (Carbone et al., 1971).

Staging was performed according to results of physical examination; surgical reports; computed tomography scan of the thorax, abdomen, and pelvis; positron emission tomography; and bone marrow biopsy. Where indicated, a bone scan, biopsies of suspicious lesions, and examination of the cerebrospinal fluid were also performed.

Lymphomas that presented in extranodal organs with no or minor lymph node involvement were considered primary extranodal, whereas lymphomas with clinically dominant lymph node involvement were considered as primary nodal. Waldeyer's ring and spleen were considered as nodal sites. The bone marrow was considered as an extranodal site.

All 100 patients were histopathologically detected as NHL. The diagnosis of NHL and its subtype was based on the criteria established in World Health Organization (WHO) classification for all cases. Immunohistochemical (IHC) analyses were performed manually on the paraffinembedded tissue sections by using a panel of monoclonal antibodies (Peroxidase-anti peroxidase method). The panel included CD45, CD20, CD3, CD15, CD30, CD5, bcl 2, cyclin D1, and anaplastic large cell lymphomakinase-1 (ALK).

The International Prognostic Index (IPI) was calculated according to the description by the international non-Hodgkin's Prognostic Factors Project for patients with all required parameters present.

Patients with CD20+, intermediate or high grade lymphoma received CHOP plus Rituximab which consisted of intravenous cyclophosphamide $(750 \mathrm{mg} /$ $\left.\mathrm{m}^{2}\right)$, intravenous doxorubicin $\left(50 \mathrm{mg} / \mathrm{m}^{2}\right)$, intravenous vincristine $\left(1.4 \mathrm{mg} / \mathrm{m}^{2}\right.$, maximum daily dose of $\left.2 \mathrm{mg}\right)$, intravenous rituximab $\left(375 \mathrm{mg} / \mathrm{m}^{2}\right)$ and per-oral tablet prednisolone (100mg D1 to D5). Patients with low grade disease or who could not receive antracycline for cardiac reasons received rituximab plus CVP or CVP. Patients with cerebral lymphoma received intravenous high-dose methotrexate $\left(4-8 \mathrm{mg} / \mathrm{m}^{2}\right)$. Patients who had bulky disease at presentation, partial response or stable disease after chemotherapy were treated by involvedfield radiotherapy at a dose of $40 \mathrm{~Gy}$ in 20 fractions in conventional fractionation over 4 weeks after completion of chemotherapy. Patients with localized disease like orbital NHL were treated by involved- field radiotherapy alone at dose of $44 \mathrm{~Gy}$ in 22 fractions in conventional fractionation over 4.5 weeks.

The initial therapy and therapeutic response [complete response (CR), partial response (PR), progressive disease (PD), stable disease (SD)] were accurately documented for each case. A CR to treatment was defined by the disappearance of all clinical evidence of the disease and normalization of all laboratory values and including radiography results which were abnormal before treatment. Treatment outcome was measured using overall survival. It was measured as the interval between the beginning of treatment and death or the date of the last follow-up evaluation. Patients were usually seen every 3 months in the first two year, every 6 months in the third and fourth years and a year in subsequent years.

\section{Statistical analysis}

Data analysis was performed using the SPSS for Windows 11.5 program. Descriptive statistical analyses for continuous and cross sectional variables were expressed as mean \pm standard deviation, whereas categorical variable were expressed as number of cases and percentage. Kaplan-Meier survival analysis was used to evaluate the statistical significant effect of residential areas on overall survival, using the Log-Rank test.

\section{Results}

Primary extranodal NHL, constituted $12.4 \%$ (100 / 802) of all NHL studied during this period. Median age of the patients was 56 years (range 17-87 years) and the male: female distribution was 3:2. Ten out of 100 patients (10\%) had B-symptoms (significant fever $>380 \mathrm{C}$, drenching night sweats, and unexplained weight loss exceeding $10 \%$ of normal body weight within the last 6 months), and 6 out of the 100 patients $(6 \%)$ had elevated serum LDH levels ( $>450 \mathrm{U} / \mathrm{L}$ ) at presentation. Percentages of Stage I, II, III and IV patients were found as 52,33,9, and $6 \%$ in whole group, respectively.

Patients were also categorized according to the International Prognostic Index (IPI) for aggressive lymphoma, and the International Prognostic Index (FILIPI) for patients with follicular lymphoma according to the description by the International non-Hodgkin's Prognostic Factors Project. Low, low-intermediate, highintermediate, and high IPI were found in 76, 19, 4, and $1 \%$ of patients, respectively.

Eighty-five of 100 patients $(85 \%)$ were in stage I/ II, $9 / 100(9 \%)$ in stage III, whereas $6 / 100(6 \%)$ were in stage IV.

Head and neck lymphomas (HNL) constituted the most common site of pENL $(51 / 100,51 \%)$, followed by gastrointestinal lymphomas (GIL) $(37 / 100,37 \%)$, and cerebral lymphomas ( CL) $(5 / 100,5 \%)$.

Immunohistochemical evaluation demonstrated that $83 \%$ of pENL had B-cell immunophenotype whereas $\mathrm{T}$ cell phenotype was observed in only $7 \%$ of patients. Indeed, Diffuse large B cell Lymphoma (DLBCL) was 
the most common histological type observed in $53 \%$ of patients. Marginal zone extranodal lymphoma, follicular lymphoma, mantle cell lymphoma, MALToma, nonspecified B-cell Lymphoma and anaplastic large cell lymphoma were found in $13,7,6,6,8$, and $4 \%$ of patients, respectively.

Chemotherapy was the mainstay of treatment. Out of 100 patients, $82(82 \%)$ received chemotherapy. Forty-four patients received chemotherapy with $\mathrm{R}-\mathrm{CHOP}$ regimen, while nine patients received R-CVP regimen. The CHOP regimen (without Rituximab) was given to eight patients. In total, 27 out of 100 patients $(27 \%)$ of pENL required invoved-field radiotherapy (IFRT). Four patients were treated only by IFRT alone and in the remaining 23 patients IFRT was added to chemotherapy. Treatment modalities are shown in Table 1.

Ninety-three patients were evaluated for response to treatment. Two of these patients refused treatment, three patients underwent tonsillectomy due to lymphoma of the tonsils, one patient was operated on due to lymphoma of the parotid gland, whereas one underwent surgery due to orbital lymphoma. No postoperative treatment was given to these patients because of the low-grade nature of their pathology results. In the remaining 93 patients, after induction treatment, CR were achieved in 71 patients, PR was achieved in 11 patients, SD was achieved in 2 patients while PD was achieved in 9 patients. Nineteen patients died at the end of the study, whereas 81 patients were reported to be alive. Treatment outcome of patients according to primary site are shown in Table 2.

The median follow-up duration to the time of progression was reported as 8 months (range, 4.5-52

Table 1. Treatment Received by Patients

\begin{tabular}{lc}
\hline Treatment modality & $\mathrm{n}=100$ \\
\hline CHOP & $8 \%$ \\
MTX +RT & $3 \%$ \\
R-CHOP & $44 \%$ \\
R-CHOP+IFRT & $24 \%$ \\
R-CVP & $9 \%$ \\
Only IFRT & $4 \%$ \\
No treatment & $8 \%$
\end{tabular}

CHOP: Inj. cyclophosphamide $\left(750 \mathrm{mg} / \mathrm{m}^{2}\right)$, inj. doxorubicin $\left(50 \mathrm{mg} / \mathrm{m}^{2}\right)$ inj. vincristin $\left(1.4 \mathrm{mg} / \mathrm{m}^{2}\right.$, maximum daily döşe of $2 \mathrm{mg}$, and per oral tab. prednisolone(100 mg D1 to D5), CVP:Inj. cyclophosphamide $\left(750 \mathrm{mg} / \mathrm{m}^{2}\right)$, inj. vincristin $\left(1.4 \mathrm{mg} / \mathrm{m}^{2}\right.$, maximum daily döse $\left.2 \mathrm{mg}\right)$ and per oral tab. Prednisolone (100 mg D1 to D5), MTX: inj. methotraxete (4-6 mg/m²), R: inj. rituximab (375 $\mathrm{mg} / \mathrm{m}^{2}$ ), IFRT: involved field radiotherapy, RT: radiotherapy

Tablo 2. Treatment Outcome of Patients According to Primary Site

\begin{tabular}{lrrrrrrr}
\hline Primary Site & CR & PR & SD & PD & $\begin{array}{c}\text { No Alive } \\
\text { treatment }\end{array}$ & Exitus \\
\hline Gastrointestinal & 31 & 1 & 2 & 2 & 1 & 31 & 6 \\
Head and Neck & 34 & 6 & - & 4 & 6 & 43 & 7 \\
Cerebral & 1 & 2 & - & 2 & - & 1 & 4 \\
Breast & 1 & - & - & - & - & 1 & - \\
Testis & - & 1 & - & 1 & - & - & 2 \\
Urinary Bladder & 1 & - & - & - & - & 1 & - \\
Ovarian & 1 & - & - & - & - & 1 & - \\
Lung & 1 & - & - & - & - & 1 & - \\
ThyroidPericardTotal & $1-71$ & -111 & -2 & -9 & --7 & 1181 & -19 \\
\hline
\end{tabular}

CR:complete response, PR: partial response, SD: stable disease, PD: progressive disease

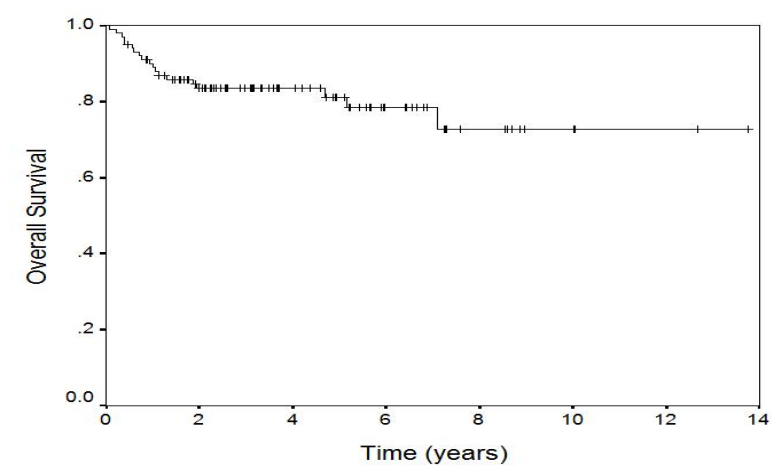

Figure 1. The Mean Survival Duration of the Patients

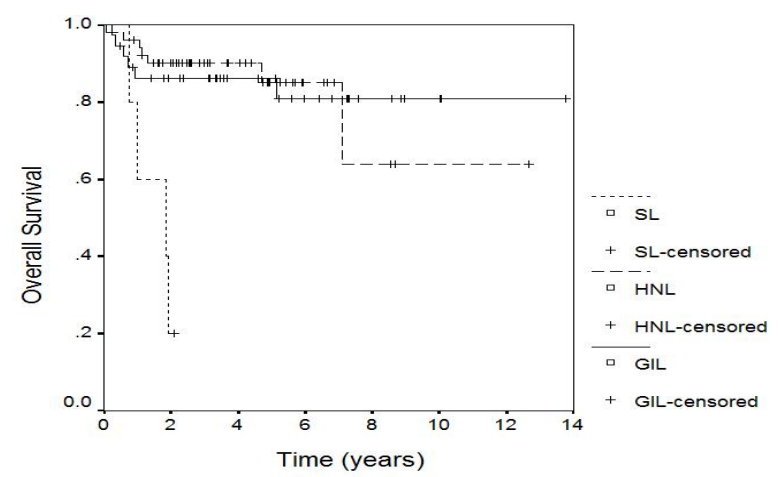

Figure 2. The Mean Survival Duration of Patients According to Site of Involvement

months), in patients (9\%) with progressive disease. The median follow-up duration of all patients was reported as 37.6 months (range, 0.8-165 months). On the other hand, the mean survival duration was found to be 129.5 months (range, 44.7-114.3 months) for the whole group; this period was reported as 137.5 months (range, 117.51578.6 months) in gastrointestinal lymphoma (GIL) patients, 119.0 months (range, 91.8-146.1 months) in head and neck lymphoma ( HNL) patients, and 18.4 months (range, 12.6-24.1 months) in cerebral lymphoma (CL) patients. The mean survival duration of the whole group and the mean survival duration of patients according to site of involvement are shown in the Kaplan-Meier curves of Figure 1 and Figure 2.

\section{Discussion}

ENL is a heterogeneous disease with regards to geographical, ethnic, anatomic, etiological and morphological diversities (Anderson et al., 1998). The frequency of ENL varies in different part of the world. Newton et al. (1997) described the epidemiology and geographical distribution of NHL in different countries. The authors used site-specific information of NHL from thirty-nine cancer centers in fourteen countries. The incidence rate for all NHL, combined varied from a low 2 per 100.000 per year in Thailand to about 10 per 100.000 in Caucasians in the USA. Across Europe, there was roughly a 2-fold difference observed from about 4 per 100.000 in Slovakia to about 8 per 100.000 in the Netherlands.

The total proportion classified as being of extranodal origin ranged from to $22-25 \%$ of all lymphomas in the USA to $33 \%$ in Denmark and 34\% in Israel. France 
(42\%) and Kuwait (43\%) had particularly high relative extranodal lymphoma frequencies (Newton et al., 1997). Compared to these epidemiological data, results of our series is very low (12.4\%). This may be explained by overall small number of NHL during the study period (802 in 10 years), or strict inclusion criteria.

ENL's may occur in any organ. Previous studies have demonstrated that the gastrointestinal tract is the most common site of involvement for ELN's, and its incidence is rising throughout the world (Arora et al., 1998). However, head and neck region including Waldeyer's ring, nose, and paranasal sinuses, have been reported to be the most common sites of origin of $\mathrm{pENL}$, in various studies from different parts of China (Yang et al., 2011), India (Singh et al., 2003), and Japan (Fujita et al., 2009). In our study, the most common site of ENL was reported for head and neck lymphomas (51\%), followed by gastrointestinal lymphomas (37\%). In variety Asian studies (Singh et al., 2003; Fujita et al., 2009; Chen et al., 2010; Yang et al., 2011), it has been shown that head and neck lymphoma are the most commonly seen forms. This difference was explained by environmental pollutants, Epstein- Barr virus, agricultural pesticides (Chen et al., 2010), poor orodental hygiene, tobacco chewing (Singh et al., 2003), and more predilection of NK/T cell lymphoma phenotypes for head and neck region (Vose et al., 2008).

The pattern of primary GIL in our study was similar to the western population as well as two largest studies from India. In these two studies, similar to our study, the stomach was the most common site of involvement and DLBCL was the most common histological subtype, whereas, according to another study in Europe (Mihaljevic et al., 2006) MALT lymphoma was accounted for $70 \%$ of GIL.

In accordance with literature, DLBCL was the most common histological subtype observed, both at nodal and extranodal sites. DLBCL, follicular lymphoma, anaplastic large cell lymphoma (ALCL) and nodal marginal zone lymphoma were the four most common subtype at nodal site; whereas DLBCL, extranodal marginal zone lymphoma of the MALT type, and peripheral T-cell lymphoma were most common at extranodal sites (Fujita et al., 2009; Yaqo et al., 2011; Yang et al., 2011). According to our study, the most common histologic subtypes of ENL were DLBCL, extranodal marginal zone lymphoma and follicular lymphoma. These results also support those in the literature.

The biological behavior of primary ENL has been reported to be complex in different studies. Krol et al. (Krol et al., 2003) showed that ENL patients had a better survival outcome, whereas, Lal et al. (2008) reported that nodal and extranodal sites did not have any prognostic significance and that the overall survival in both groups largely depended on IPI and not on the site of origin of malignancy. According to our study, patients with pENL had lesser B symptoms, lower serum LDH level, a high percentage of low and low-intermediate IPI score (95\%) and high in the early stage (stage 1 and stage 2,81\%). These results probably explained the favorable prognosis in our cohort of patients.

In conclusion, according to our study, the incidence of pENL in Turkey's southern region is low, compared to data from other parts of the world. Head and neck, and the gastrointestinal tract were the two most common extranodal sites observed. Histologically DLBC accounted for the majority of cases. Most patients were on earlier stages, had low-low intermediate IPI scores and had a favorable prognosis. Future efforts should focus on genetic profile of lymphomas in order to understand the biology of this group of tumors.

\section{References}

Anderson JR, Armitage JO, Weisenburger DD (1998). Epidemiology of the non-Hodgkin's lymphomas: distributions of the major subtypes differ by geographic locations. Non-Hodgkin's Lymphoma Classification Project. Ann Oncol, 9, 717-20.

Arora N, Manipadam MT, Pulimood A, et al (1998). Gastrointestinal lymphomas: Pattern of distribution and histological subtypes: 10 years' experience in a tertiary centre in South India. Ind J Pathol Microbiol, 54, 712-9.

Carbone PP, Kaplan HS, Musshoff K, et al (1971). Report of the Committee on Hodgkin!s disease Staging Classification. Cancer Res, 31, 1860-1.

Chen W, Tsai W, Chao T (2010). The clinicopathological analysis of 303 cases with malignant lymphoma classified according to the World Health Organization classification system in a single institute of Taiwan. Ann Hematol, 89, 553-62.

Chiu BC, Weisenburger DD (2003). An update of the epidemiology of non-Hodgkin's lymphoma. Clin Lymphoma, 4, 161-8.

d'Amore F, Christensen BE, Brincker H, et al (1991). Clinicopathological features and prognostic factors in extranodal non-Hodgkin lymphomas. Danish LYFO Study Group. Eur J Cancer, 27, 1201-8.

Dawson IP, Cornes JS, Morson BC (1961). Primary malignant lymphoid tumours of the intestinal tract. Report of 37 cases with a study of factors influencing prognosis. Br J Surg, 49, 80-9.

Freeman C, Berg JW, Cutler SJ (1972). Occurrence and prognosis of extranodal lymphomas. Cancer, 29, 252-60.

Fujita A, Tomita N, Fujita H, et al (2009). Features of primary extranodal lymphoma in Kanagawa, a human T-cell leukemia virüs type 1 nonendemic area in Japan. Med Oncol, 26, 49-54.

Groves FD, Linet MS, Travis LB, et al (2000). Cancer surveillance series: Non-Hodgkin's lymphoma incidence by histologic subtype in the United States from 1978 through 1995. J Natl Cancer Inst, 92, 1240-51.

Krol AD, le Cessie S, Snijder S, et al (2003). Primary extranodal non-Hodgkin's lymphoma (NHL): the impact of alternative definitions tested in the Comprehensive Cancer Centre West populatin-based NHL registry. Ann Oncol, 14, 131-9.

Lal A, Bhurgri Y, Vaziri I, et al (2008). Extranodal non-Hodgkin's lymphomas- a retrospective review of clinico-pathologic features and outcomes in comparison with nodal nonHodgkin's lymphomas. Asian Pac J Cancer Prev, 9, 453-8.

Mihaljevic B, Jancic-Nedeljkov R, Vujicic V, et al (2006). Primary extranodal lymphomas of gastrointestinal localizations: a single institution 5-yr experience. Med Oncol, 6, 23, 225-35.

Newton R, Ferlay J, Beral V, Devesa SS (1997). The epidemiology of non-Hodgkin's lymphoma: comparison of nodal and extranodal sites. Int J Cancer, 72, 923-30.

Otter R, Gerrits WB, vd Sandt MM, et al (1989). Primary nodal and extranodal non-Hodgkin's lymphoma. A survey of a 

population-based registry. Eur J Cancer Clin Oncol, 25, 1203-10.

Padhi S, Paul TR, Challa S, et al (2012). Primary extra nodal non Hodgkin lymphoma: a 5 year retrospective analysis. Asian Pac J Cancer Prev, 13, 4889-95

Singh D, Kumar L, Goyal H (2003). Primary extranodal nonHodgkin's lymphoma in northern India. Proc Am Soc Clin Oncol, 22, 2457.

Vose J, Armitage J, Weisenburger D (2008). International T-cell lymphoma project. international peripheral t-cell and natural killer/T-cell lymphoma study: pathology findings and clinical outcomes. J Clin Oncol, 26, 4124-30.

Yang QP, Zhang WY, Yu JB, et al (2011). Subtype distribution of lymphomas in southwest china: analysis of 6,382 cases using WHO classification in a single institution. Diagnostic Pathology, 6, 77.

Yaqo RT, Hughson Md, Sulayvani FK, et al (2011). Malignant lymphoma in northern Iraq: A retrospective analysis of 270 cases according to the world health organization classification. Ind J Cancer, 48, 446-51. 